\title{
Investigation of large scale shock movement in transonic flow
}

\author{
C. Wollblad $^{1}$, L. Davidson ${ }^{* 2}$, L.-E. Eriksson ${ }^{3}$ \\ Division of Fluid Dynamics, Department of Applied Mechanics, Chalmers University of \\ Technology, SE-41296, Gothenburg, Sweden
}

\begin{abstract}
Large eddy simulations were made of transonic flow over a two-dimensional bump where shock wave turbulent boundary layer interaction takes place. Different flow conditions were investigated to find conditions for large scale shock movement. The innermost part of the shock was found to be moving for sufficiently strong shocks. None of the cases display large scale movement of the whole shock.
\end{abstract}

Key words: Transonic flow, LES, shock wave/turbulent boundary layer interraction

\section{Introduction}

Shock wave turbulent boundary layer interactions (SWTBLI) have been studied for many years. These situations commonly arise in turbo machinery and aerospace applications and on the exterior of high speed aircraft. In all these cases, shock wave boundary layer interaction can significantly change the flow and hence the physical load imposed by it. The performance of the object studied can also be significantly altered if shocks and boundary layer separations do not occur where expected.

\footnotetext{
*Corresponding author

Email address: lada@chalmers.se (L. Davidson)

${ }^{1} \mathrm{PhD}$ student.

${ }^{2}$ Professor in Heat Transfer.

${ }^{3}$ Professor in Compressible Flow.
} 
This paper is a continuation of previous work in which a transonic flow with SWTBLI was calculated by large eddy simulation (LES) (Wollblad et al., 2006). There the authors found a particular configuration for which the known prerequisites for large scale shock movement were fulfilled (AGARD, 1986; Wu and Martin, 2004) but where there was no such movement. This configuration, hereafter denoted the baseline case, and some of its characteristics are described in section 2.

Several other investigations have been made of both transonic and supersonic SWTBLI. Some will be discussed in section 3 and their configurations will be used to motivate alterations of the well documented case in Wollblad et al. (2006). The motivation is to find more precise conditions for large scale shock movement in transonic SWTBLI.

\section{The baseline case}

\subsection{Computational set-up}

The computational domain is a numerical model of a part of an experimental test rig at the Department of Energy Technology, KTH. The experimental test section is $0.44 \mathrm{~m}$ long, $0.10 \mathrm{~m}$ wide and $0.12 \mathrm{~m}$ high. There is a bump at the bottom of the test section. See Bron (2003) for greater detail about the rig. Our domain consists of this test section but has been shortened by $0.08 \mathrm{~m}$ and translational periodicity was assumed in the spanwise direction for a width less than that of the test section. Previous numerical investigations have shown that a width of $0.039 \mathrm{~m}$ is sufficient to let the flow develop unhindered by a limited spanwise domain. Furthermore, the ceiling was removed and replaced by a symmetry plane. A $2 D$ picture of the domain is shown in figure 1. The parameters in the baseline case were selected to mimic the conditions in the test rig as far as possible. Hence, the symmetry plane was lowered by one momentum loss thickness based on the inlet boundary condition, so that $h=0.1186 \mathrm{~m}$. In this way possible differences in mass displacement between measurements and calculations were reduced. The maximum height of the bump is $0.01048 \mathrm{~m}$.

The geometry is such that, for some subsonic boundary conditions at the inlet and outlet, the flow will be accelerated over the bump and the sonic pocket that is formed will be terminated by a shock.

To ensure a well resolved LES of the case, the Reynolds number was reduced by a factor of 11.25 compared to the experiments. This was done by increasing the dynamic viscosity to $\mu=1.8 e^{-4} \mathrm{Pas}$. The inlet boundary 


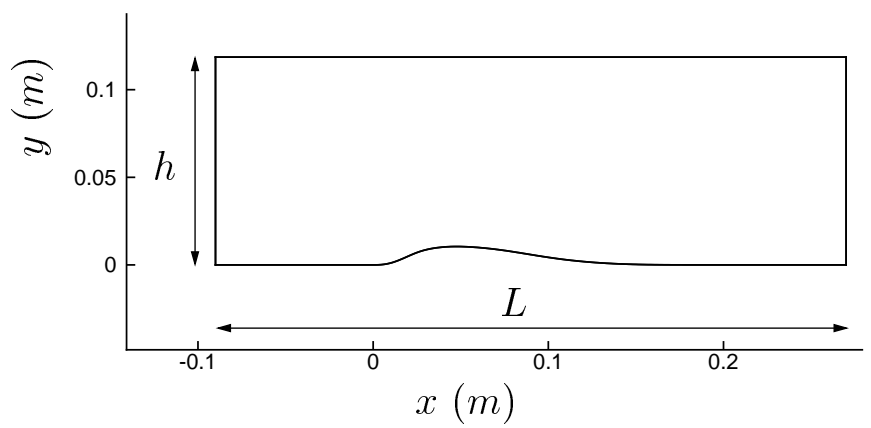

Figure 1: $2 D$ projection of the computational domain.

conditions had freestream values of $M=0.70, P_{0}=160.0 \mathrm{kPa}, T_{0}=303 \mathrm{~K}$ and the boundary layer fluctuations were taken from incompressible DNS of fully developed channel flow. The Reynolds number based on $u_{*}$ and $\delta_{99}$ at the inlet was 632 and the momentum dislacement thickness $0.89 \mathrm{~mm}$. The outlet pressure was set to $103.5 \mathrm{kPa}$.

As mentioned, the upper boundary was set to be a symmetry boundary and the spanwise boundaries were given translational periodic boundary conditions. No-slip, adiabatic conditions were enforced along the wall.

\subsection{Large Eddy Simulation Numerics}

The conservative forms of the continuity, momentum and energy equations were solved on structured meshed using the finite volume method. The larage eddy formulation is obtained using a box fileter of grid cell size. Code documentation can be found in Eriksson (1995).

The inviscid fluxes are discretized in space using a fourth order central scheme to which is added a small amount of upwinding. The upwinding is a third difference multiplied by a user defined constant and applied to the characteristic variables, which are in turn evaluated using a planar wave assumption. This construction makes the space discretization of the inviscid fluxes third order accurate in terms of dissipation, but still makes it fourth order in terms of dispersion.

A second order central scheme is used for the viscous fluxes. The subgrid terms were modeled using a compressible version of the WALE model developed by Nicoud and Ducros (1999). The modification is described in 
Wollblad et al. (2006). The filter width was calculated by $(\Delta x \Delta y \Delta z)^{1 / 3}$, i. e. to a function of the grid.

At strong pressure gradients, such as in the case of shocks, extra diffusion is needed to prevent the calculations from diverging. Here a term scaled by the second derivate of the local pressure and the spectral radius of the discretized flux operator is added in the calculations of the inviscid fluxes (Jameson et al., 1981). The user defined coefficient that guides the level of extra dissipation is set to a level just high enough to prevent divergence, which in this work is twice the value used in Wollblad et al. (2006).

The code uses a three-stage, second-order accurate, low storage RungeKutta method. Close to walls, a semi-implicit preconditioning scheme is applied in each step of the Runge-Kutta method. The semi-implicit method is described in detail in Wollblad et al. (2004).

For further details on the numerical treatment, see Wollblad et al. (2006) and references therein.

\subsection{Data Treatment}

A calculation was run until the average wall shear stress on the back of the bump showed no long time fluctuations. The calculations were run for approximately six flow-through times. During this time 850 instantaneous solutions with equal time spacing were saved. Tests showed that second order statistics could be calculated from only 500 samples with no loss of accuracy, but it was decided that 850 samples should be used so that there would also be accuracy in the computations of time-space correlations.

The time series are not long enough to obtain well resolved spectra. The spectra have however been calculated using only 600 of the time steps. The result was that the peaks changed somewhat in magnutide, but the important frequencies remained the same.

The code gives data in the form of cell averages. For each node (node $=$ grid node), the values of the adjacent cells were added and the sum was divided by the number of adjacent cells. This makes the values of the interior nodes averages of eight cell values, while the values assigned to a corner node equal those of its adjacent cell.

Depending on the application, the nodal data were then either averaged in the spanwise direction or interpolated onto the cells of another grid. Flow statistics such as Reynolds stresses were calculated from data averaged in both the spanwise direction and in time. For calculation of correlations and frequencies, data were interpolated to a sample grid and calculations carried 


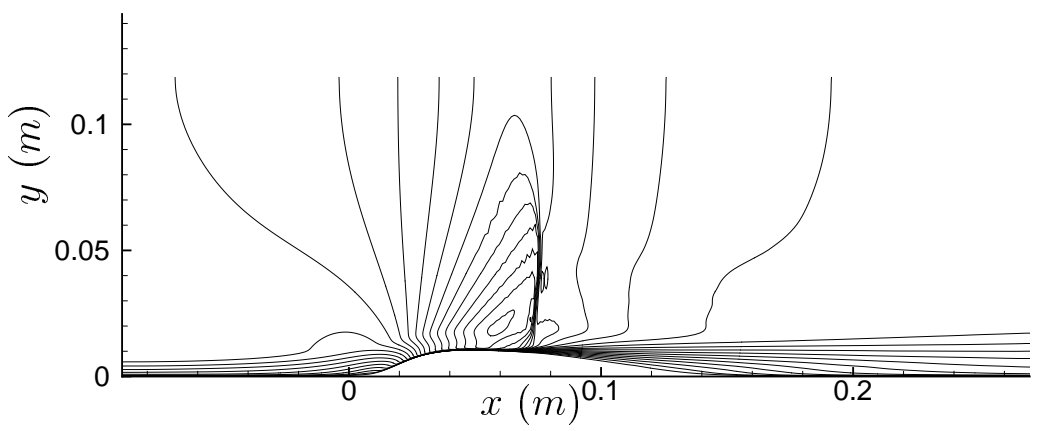

Figure 2: Mach number contour plot for the baseline case. The inlet free stream Mach number is 0.7 and maximum Mach number before the shock is 1.27 . The contour level difference is 0.05 .

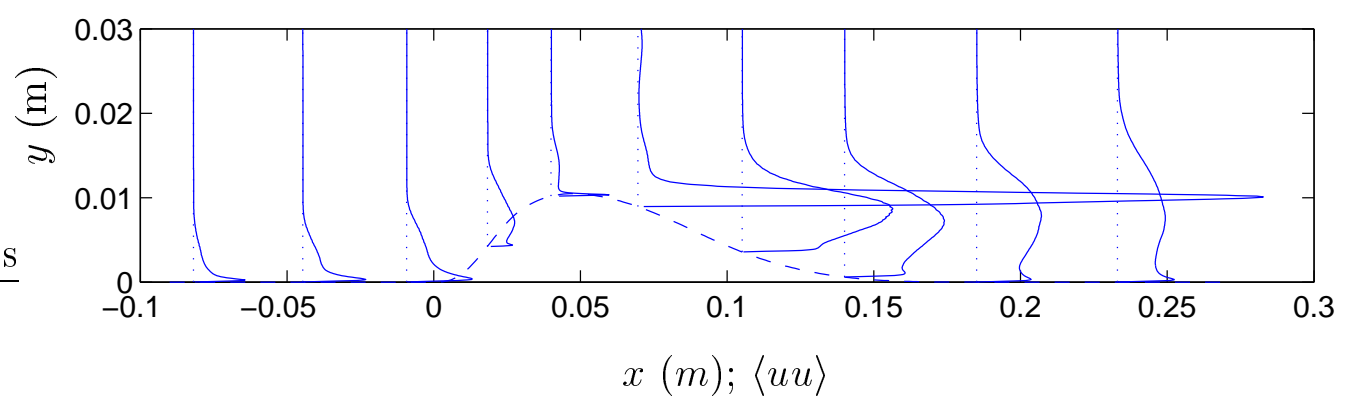

Figure 3: Resolved $\langle u u\rangle$ Reynolds stresses of the baseline case.

out on this grid using the cell values. The results were then averaged in the spanwise direction.

\subsection{Flow characteristics of the baseline case}

This subsection summarizes the results of the simulations reported in Wollblad et al. (2006). The Mach number contour plot in figure 2 shows the general picture of the mean flow. The flow accelerates to form a sonic pocket that is terminated by a shock at $x \approx 0.075 \mathrm{~m}$. The maximum Mach number before the shock is 1.27. The Mach number gradient through the shock is very steep, which indicates that the shock is absolutely stable. This is confirmed both by FFT analysis of the shock position and by visualization of the flow. The exception is the innermost part of the shock, but the movement is more a variation in strength caused by incoming turbulent structures in the 
boundary layer. FFT of the innermost shock position reveals no dominating frequency.

The streamwise resolved Reynolds stresses (scaled for maximum visability) are shown in figure 3 . Observe that the $y$-scale is different from that in figure 2. The boundary layer develops as expected without any strange initial transients. As the fluid accelerates at the front of the bump, the favorable pressure gradient starts a relaminarization process. The boundary layer remains however turbulent for all $x$. This relaminarization process is the most notable Reynolds number dependent part of the flow since the process is directly proportional to the viscosity (Jones and Launder, 1971). As the flow separates, the resolved $\langle u u\rangle$ Reynolds stresses are amplified as much as $300 \%$ and the flow becomes highly anisotropic. The separated boundary layer continues more or less horizontally while a new boundary layer forms downstream of the shock.

The separation characteristics are demonstrated in figure 4 where the probability density function (PDF) of the skin friction coefficient is shown as a function of the streamwise position. As can be seen, the interaction region, i. e. the region where the effect of the shock is felt, starts at $x \approx 0.045 \mathrm{~m}$ and the flow separates at $x \approx 0.06 \mathrm{~m}$. The probability of back flow can be found by integrating the PDF for all negative $C_{f}$; the result is shown in figure 5 . Clearly, there is a separated region, $P\left(C_{f}<0\right)>0.5$, of $3 \mathrm{~cm}$ ranging from $x \approx 0.06 m$ to $x \approx 0.09 \mathrm{~m}$. Three centimeters is in this context a rather large scale and hence the flow can be regarded as strongly separated.

The lack of large scale shock movement is in disagreement with many experiments of transonic SWTBLI, for example those presented by Bron (2003), but in agreement with LES presented by Sandham et al. (2003) and experiments presented by Bachalo and Johnson (1986).

The connection between flow structures and unsteady shock movement has chiefly been investigated for supersonic flow in compression ramps. Thomas et al. (1994) related the shock movement to the frequency of the separated region. There are significant differences between the current case and a supersonic compression ramp. In the supersonic case, the separation bubble is 'trapped' between the shock and the compression ramp, whereas the current flow features not one but several separation bubbles. Thus, no single turnover time can be computed for the current case, although the analogy can still be useful. On the other hand, several investigations of supersonic compression ramp flow have pointed out the bursting frequency of the incoming boundary layer to be the frequency of the shock movement. See for example Wu and 


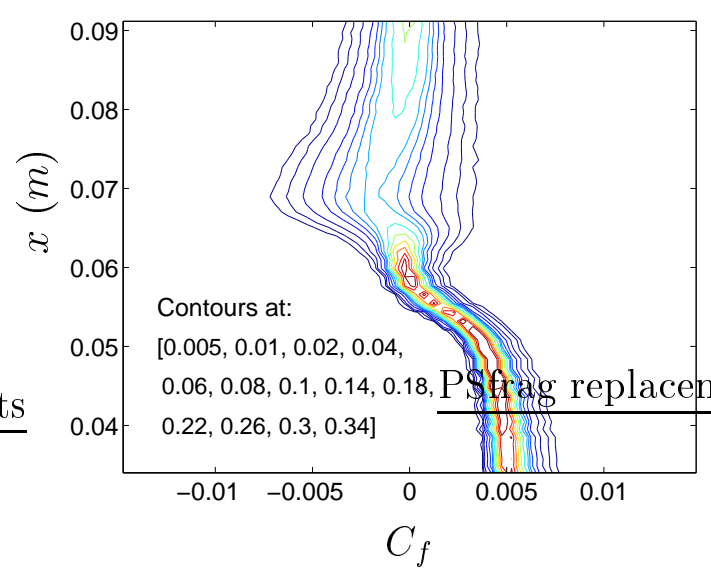

Figure 4: Contour lines of the PDF of $C_{f}$

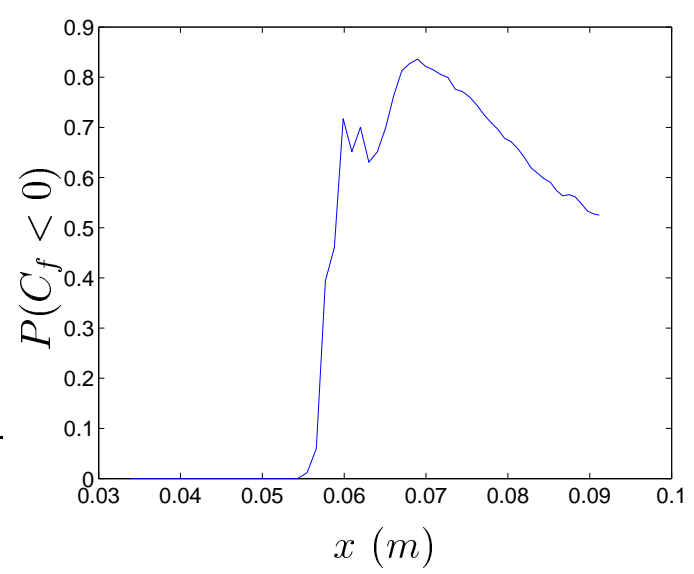

Figure 5: The probability for $C_{f}$ to be less than zero as a function of the streamwise position.

Martin (2004) and Andreopoulos and Muck (1987). They found that bursts and shock movement both had a main frequency of $0.14 U_{\infty} / \delta$ which for the baseline case corresponds to $5.8 \mathrm{kHz}$ at $x=0.05 \mathrm{~m}$. The baseline case does indeed feature bursting events with a main frequency of $5.5 \mathrm{kHz}$ at that position (Wollblad et al., 2006). This indicates that bursting events in the incoming boundary layer do not trigger large scale shock movement in the transonic case.

\section{Comparison with other studies}

The most common configuration for studying transonic SWTBLI is the supercritical airfoil, such as the one shown in figure 6. Shock movement in such a configuration is known as "the buffeting phenomenon". Recent numerical investigations (Deck, 2005; Xiao and Tsai, 2006) support the theory proposed by Lee (1990) who suggested that the separation (point 2 in figure 6 ) causes large scale structures that are convected downstream. As these structures reach the trailing edge (point 1 in figure 6) they interact with the flow coming from underneath the airfoil. This interaction causes a radiation of acoustic waves which in turn propagate upstream to the shock, leading to a feedback mechanism.

Since the current geometry does not have a trailing edge, no feedback mechanism such as that in the airfoil case can be present. It is however in- 


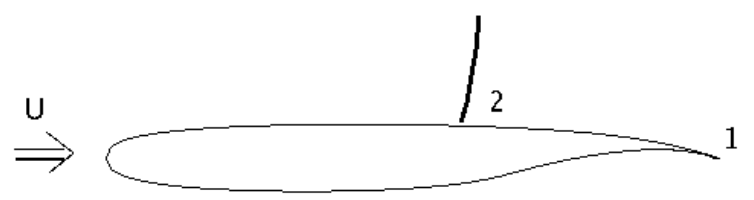

Figure 6: Whitcomb supercritical airfoil. Flow from left to right. $U$ is the free stream velocity. 2 indicates the separation region behind the shock.

teresting to note that it, in the case of the wing, is waves upstream traveling from far downstream of the shock that determine the large scale shock movement rather than bursting events in the incoming boundary layer or direct interaction between the shock and the separation bubble directly behind the shock.

The airfoil discussion indicates that the necessary conditions for large scale shock movement in internal transonic flow must probably be sought elsewhere than at the shock foot. One common feature in most such flows is that they are choked. This can be seen in many pictures in AGARD (1986) and in the cases reported by Handa et al. (2003) and Moroianu (2003). The hypothesis that choking is necessary is supported by the work of Bachalo and Johnson (1986) who considered an unchoked axisymmetric configuration and observed that the inviscid flow on their model was "exceptionally stable". Choking is however probably not a sufficient condition as can be seen in the work of Sandham et al. (2003) who reported LES of a flow very similar to the baseline case. The differences are that the bump geometry in that case is the top of a cylinder, their shock covers the whole domain up to the symmetry plane and the maximum Mach number before the shock is 1.16. The shock is stable however, exactly as in the baseline case. The contradiction to the hypothesis that choking is a necessary condition is the measurements made by Bron (2003) where large scale shock movement is reported even though the flow is not choked.

The possible connection between large scale shock movement and a choked internal flow obviously needs further investigation. A case in which the symmetry plane of the baseline case has been lowered to $y=0.08 \mathrm{~m}$ while all other flow parameters are kept constant will be presented. Another case 


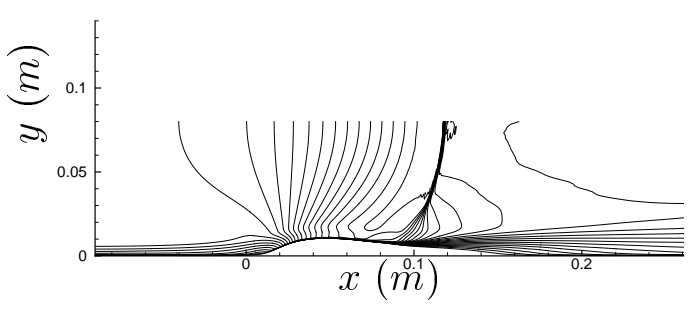

(a) Case 1

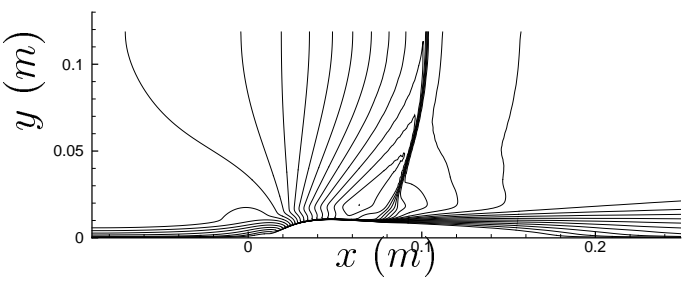

(b) Case 2

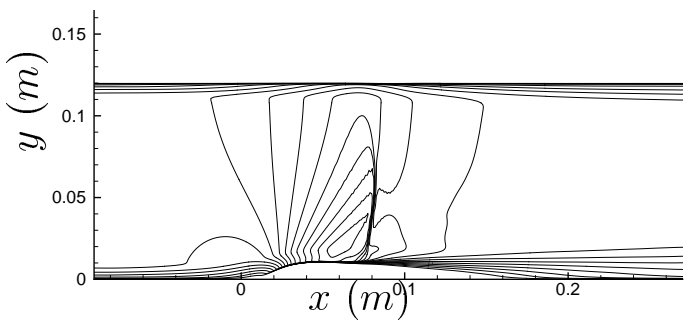

(c) Case 3

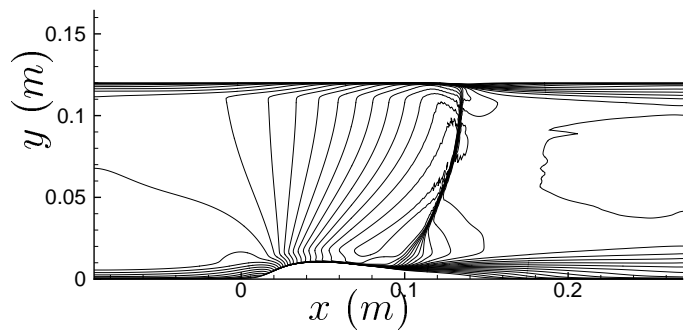

(d) Case 4

Figure 7: Mach number contour plots. The inlet free stream Mach number is 0.7 and the contour level difference is 0.05 . Maximum Mach number before the shock is (a) 1.4, (b) 1.3, (c) 1.28, (d) 1.38 . 


\begin{tabular}{|l|l|l|l|}
\hline \hline Case & Domain hight & Outlet pressure & Ceiling \\
\hline baseline & $0.1186 \mathrm{~m}$ & $103.5 \mathrm{kPa}$ & no \\
\hline 1 & $0.08 \mathrm{~m}$ & $103.5 \mathrm{kPa}$ & no \\
\hline 2 & $0.1186 \mathrm{~m}$ & $101 \mathrm{kPa}$ & no \\
\hline 3 & $0.12 \mathrm{~m}$ & $101 \mathrm{kPa}$ & yes \\
\hline 4 & $0.12 \mathrm{~m}$ & $96 \mathrm{kPa}$ & yes \\
\hline \hline
\end{tabular}

Table 1: Summary of the cases.

where the geometry is the same as in the baseline case but where the outlet pressure is lowered will also be presented. Both alterations will create choked flows with stronger shocks than in the baseline case. The case with lower ceiling will experience a relatively larger degree of blocking since the bump will cover a relatively larger part of the domain. This can be significant for eventual large scale shock movement. Sajben and Kroutil (1981), who investigated the flow in a transonic diffuser, reported increased shock movement if the blockage was increased by alterating the boundary layer before the throat.

A common approach, especially for LES, is to replace the ceiling of the channel with a symmetry plane. Handa et al. (2003) made experiments and quasi one-dimensional computations of a transonic diffuser. They investigated two different maximal Mach numbers before the shock (1.26 and 1.48), and the diffuser was choked. They suggest that when pressure waves generated in the separated region hit the ceiling, new pressure waves are created that travel upstream along the ceiling and move the shock as they reach it. Their hypothesis is consistent with the simulations made by Moroianu (2003) where the ceiling was included, and with the simulation made by Sandham et al. (2003), where the ceiling was replaced by a symmetry plane. Two simulations will be presented where the ceiling of the domain is included, one case in which the flow is not choked and one in which it is choked.

Table 1 summarizes the four cases that will be presented. Note that the domain height in cases 3 and 4 is restored to the same value as in the test rig at KTH. For all cases, the resolution and numerical paramters are the same as for the baseline case for which grid convergence and independence of parameters of the numerical scheme was proved in Wollblad et al. (2006). For cases 3 and 4, the ceiling boundary layer has the same resolution as the 
baseline case boundary layer has at the inlet, i.e. $\Delta x^{+} \approx 100, \Delta z^{+} \approx 15$ and $\Delta y^{+} \leq 1.0$. There is no case that includes the possible side wall effects. Side wall effects could of course be necessary to trigger large scale shock movement, but since the object is to test existing hyposethes rather than strictly reproducing the results of Bron (2003), side wall effects have been left for future investigations.

\section{Basic features}

\subsection{Case 1}

Figure 7(a) shows a Mach number contour plot of the average flow field of case 1 . The very sharp shock in the free stream region indicates what is confirmed by unsteady visualization of the flow: the shock is stable. Compared to the baseline case, the shock is located further downstream and is hence stronger and has a more sizable separation region. The maximum average Mach number before the shock is 1.4 and the location of the maximum is much further from the wall than in the baseline case.

Compared to the baseline case, the innermost part of the shock is moving much more. The position of the shock is measured by the maximum pressure gradient one centimeter above the bump floor $(y=0.018)$. The power spectra of the baseline case and case 1 are compared in figure 8(a). The standard deviation for the shock position in case 1 is $3.3 \mathrm{~mm}$ while it is only $0.34 \mathrm{~mm}$ for the baseline case. The peak at $350 \mathrm{~Hz}$ is interesting since it is one of the frequencies found in measurements of the wall pressure made by Bron (2003).

\subsection{Case 2}

The average solution of another choked configuration is shown in figure 7(b). The shock position is much more comparable with the baseline case than with case 1 and the maximum average Mach number before the shock is 1.3. It also shares the feature that the shock position is stable. Hence, cases 1 and 2 confirm that a choked computational domain is not a sufficient condition for large scale shock movement in internal transonic flows.

The standard deviation of the position of the innermost part of the shock is $2.2 \mathrm{~mm}$. The power spectrum of the movement has two dominant peaks, one at $350 \mathrm{~Hz}$ and one at $900 \mathrm{~Hz}$, both with $P_{h} \approx 4 \cdot 10^{-4}$. Case 1 has its strongest and second strongest peaks at the same frequencies (see figure $8(\mathrm{a}))$. 
As will be seen throughout the rest of this article, the separation characteristics of case 2 shares most of its features with case 1 . Hence, the degree of blockage seems to have very little effect on the type of flow considered here.

\subsection{Case 3 and 4}

Case 3 is very similar to the baseline case, i.e. strong separation but without large scale shock movements. Mach number contours are shown in figure $7(\mathrm{c})$. As can be seen, the shock does not reach the ceiling and hence the upper boundary layer does not separate. The flow is not choked, neither in mean nor instantaneously. This calculation only confirms that as long as the shock does not reach the upper boundary there is no difference in having a symmetry plane or a solid wall. The only real difference is that, to get the same flow field qualitatively, the outlet pressure must be lower if the ceiling is included since the upper boundary layer creates extra drag.

A mean Mach number plot of case 4 is shown in figure $7(d)$. The maximum Mach number before the shock is 1.38 and is hence comparable with that of case 1 . There is separation along the ceiling. The backflow region there is rather small and no large scale structures are produced by this separation.

Another difference between case 4 and cases 1 and 2 is that a much larger part of the shock is unsteady. The line of the maximum Mach number goes from $(x, y) \approx(0.08,0.02)$ up to the shock at $(x, y) \approx(0.12,0.054)$. Visualization of the flow shows that the shock above the latter point is stable while the shock beneath the point is unstable, as is the line of the maximum Mach number. As can be seen in figure $8(\mathrm{~b}), 350 \mathrm{~Hz}$ is no longer the dominating frequency.

\section{Separation characteristics}

The probability for backflow of case 1 is shown in figure 9. Backflow is defined as negative wall shear stress. Although the duality of the peak, which figure 5 gives an implication of, could be an indication of lambda shock, no lambda pattern can be found. All of the investigated cases display shocks that consist of one steep pressure gradient. In some of the cases, the innermost part of the shock does however have two preferred positions. Case 1 has this property; a histogram of the shock location in case 1 is shown in figure 10. Case 2 is the only other case beyond case 1 to display this feature. The baseline case and case 3 have virtually no shock movement, 


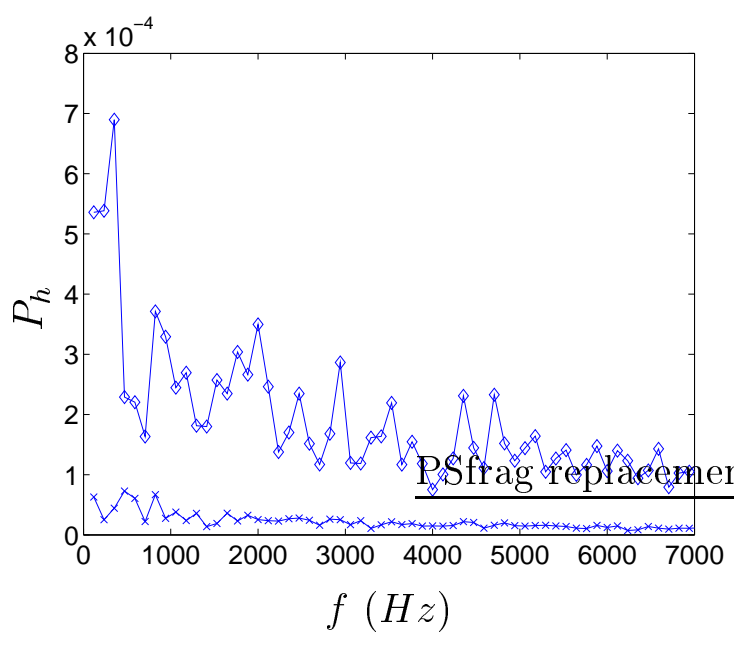

(a) Baseline case $(\times)$ and case $1(\diamond)$

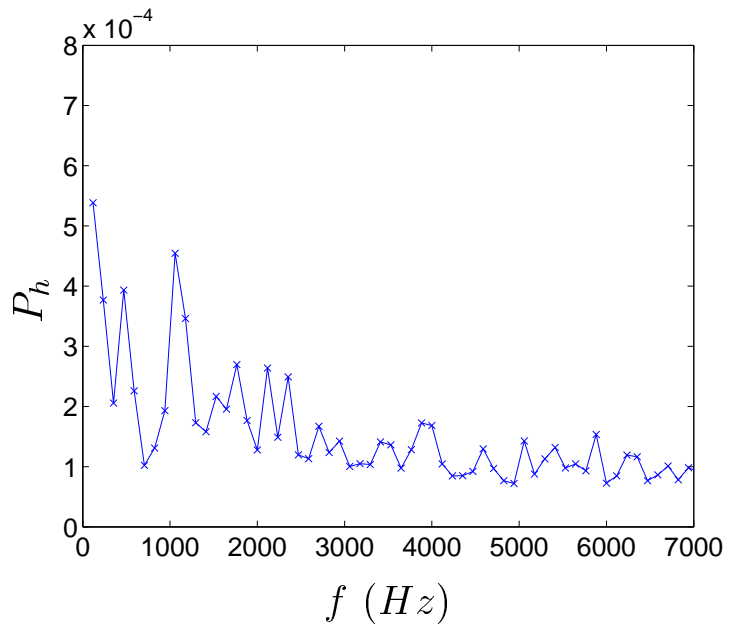

(b) Case 4

Figure 8: Power spectra of positions of the innermost parts of the shocks.

and the distribution of the shock position of case 4 is monomodial. All cases do however display a dual peak in the probability of backflow and hence this bimodality of the shock position cannot explain the dual peak of the probability of backflow.

The explanation can instead be found by closer examination of figures 4 and 5 . The position of the first peak (at $0.06 \mathrm{~m}$ ) in figure 5 can be seen to be at a location where the distribution of $C_{f}$ is still very narrow. That means that the flow there still has relatively small Reynolds stresses and that the separation is stable. The second peak $($ at $0.07 \mathrm{~m}$ ) is well within the region in which $C_{f}$ has a very broad distribution, i. e. where the Reynolds stresses are large. The corresponding streamlines are sketched in figure 11. The boundary layer separates when it approaches the shock, and a small, thin, but stable separation bubble forms. Behind the bubble, a second, much larger, separation bubble forms. There is an attached boundary layer region between the two bubbles where fluid is exchanged between the bubbles. The second separation bubble is however unstable and now and again detaches. When it does, the flow behind the first bubble temporarily reattaches, creating the dip in the probability of backflow. The separation of the second bubble is completed when a new bubble is created filling its place. This detachment of the large separation bubble explains the large Reynolds stresses. 


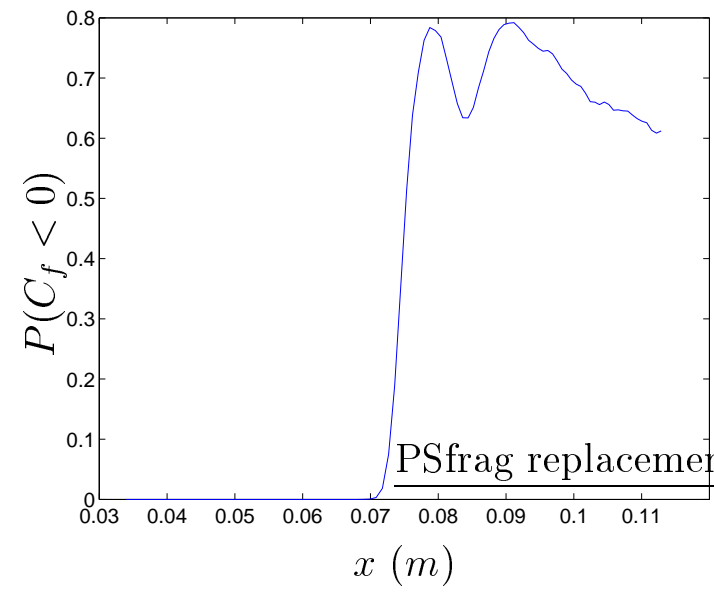

Figure 9: Contour lines of the PDF of $C_{f}($ case 1$)$.

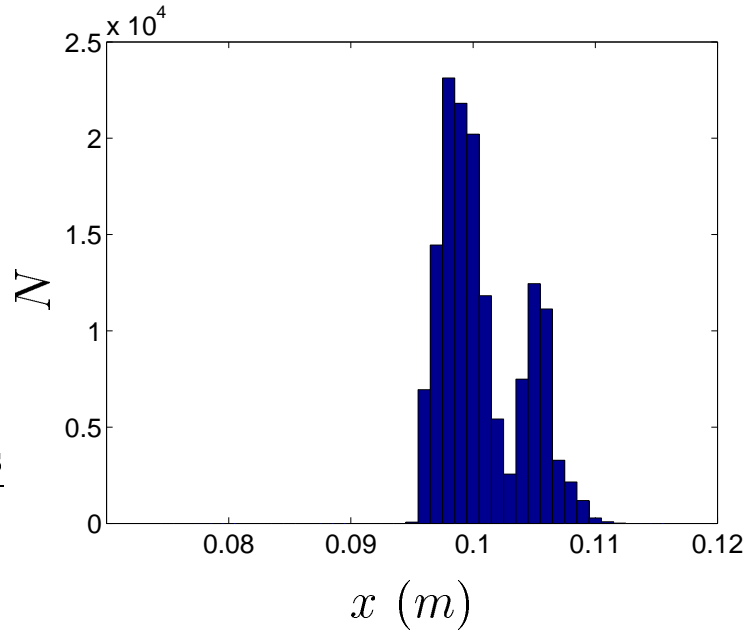

Figure 10: Histogram of the position of the inner part of the shock (case 1).

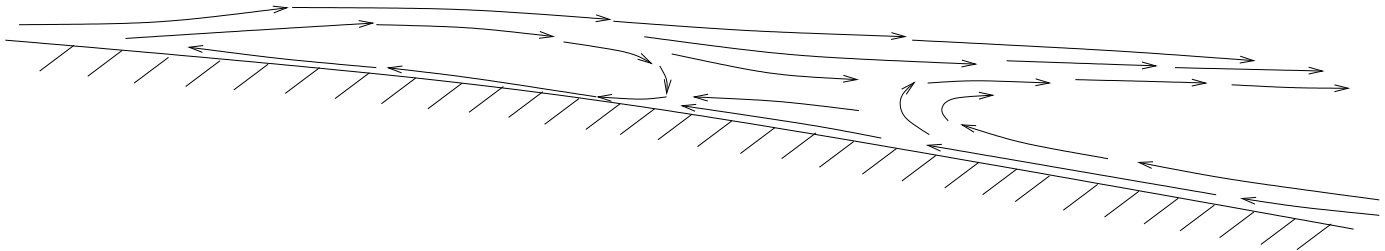

Figure 11: Sketch of streamlines at the wall beneath the shock. The height of the first separation bubble is approximately one millimeter.

Tha above conclusions have been drawn mainly from streamline animations. An example from the baseline case is however shown in 12 .

The frequency of the separation of the larger separation bubble is however not the same as the frequency of the innermost part of the shock. This can be seen by observing that the location of the local minimum of the probability of backflow in case 4 is $x=0.086$. Power spectra of the logical function $\tau_{w} \geq 0$ at that position show a dominating frequency of $2 \mathrm{kHz}$. This is the frequency of the reattachment at that point and hence also of the detachment of the larger separation bubble while the dominating frequency of the shock movement is a between $350 \mathrm{~Hz}$ and $1200 \mathrm{~Hz}$.

The separation and the shock movement can still be connected through 
elliptic leakage, i.e. information traveling upstream past the shock through the subsonic boundary layer. This hypothesis is supported by the following analysis: take a phenomenon with a high frequency and another phenomenon with a low frequency, in this case the detachment of the second separation bubble and the movement of the shock. If the faster phenomenon triggers the slower phenomenon, a time correlation between the phenomena will display peaks with a time separation of $1 / f_{s}$ where $f_{s}$ is the frequency of the slow phenomenon. Power spectrum of the correlation between the wall shear stress at $x=0.086$ and the shock position for case 4 is shown in figure 13 . It can be seen that the two most important frequencies are the same as in figure 8(b). These conclusions can also be drawn by studying time correlations made between density fluctuations in the outer part of the separated region behind the shock $(x=0.12$ and $y=0.007-0.011)$ and the shock positions.

Bibko et al. (1990) and Glotov (1998) made experimental studies of SWTBLI in compression corners. Both studies came to the conclusion that the main reason for shock wave fluctuations is the disturbances that appear in the separation region. These disturbances travel upstream through the boundary layer and trigger shock movement ${ }^{4}$. The findings here are consistent with these investigations.

${ }^{4}$ The article of Glotov is in Russian but its conclusions are summarized in Knight et al. (2003).

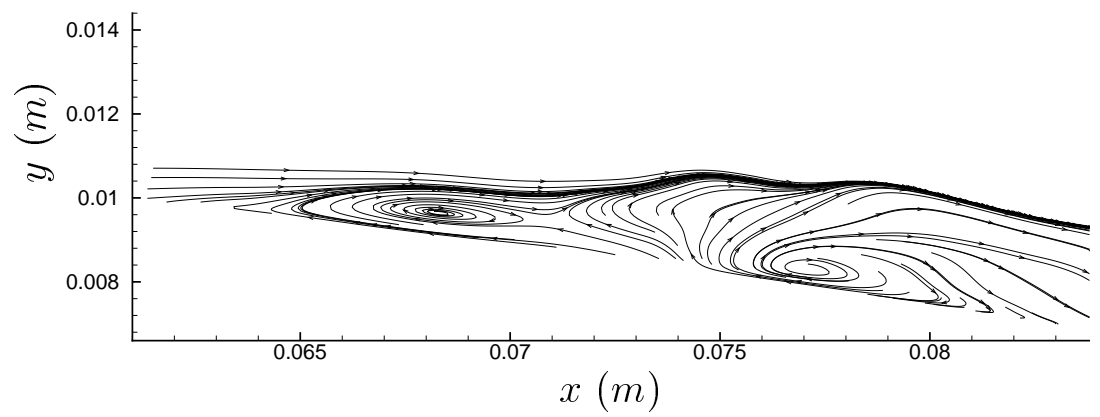

Figure 12: Streamlines from the baseline case. 


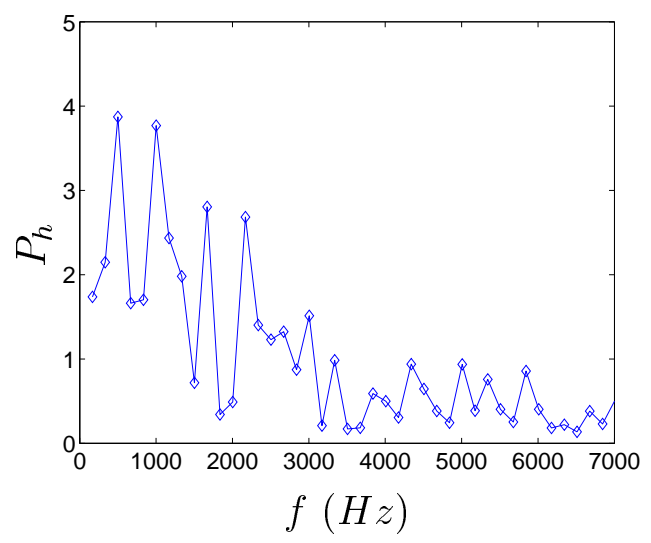

Figure 13: Power spectra of the time correlation between $c_{f}$ and the position of the innermost part of the shock. Case 4 .

\section{The path of information}

As discussed in section 3, the question of the origin of self induced large scale shock movement is strongly connected to interactions between different parts of the flow. The crux of the matter is what information that might make the whole shock move and what the origin of this information is.

A way to visualize the information transport is to plot $d \bar{p} / d t$ as shown in figure 14. The figure has labeled arrows to facilitate the discussion that follows here. Because it is a very sensitive quantity, $d \bar{p} / d t$ is extracted directly from the computational code. Case 2 is chosen for the discussion since it most clearly displays the important features. Some of the features given below are not obvious from a single snapshot but have been concluded from time dependent visualizations of $d \bar{p} / d t$.

The boundary layer at the inlet can be seen to generate a great deal of sound (arrow number 1). This is typical for a non-equilibrium boundary layer and is most prominent in transition (Bodony and Lele, 2006; Cabana et al., 2006). The boundary layer does however quickly reach a near-equilibrium state and there is a much weaker generation of noise when the flow reaches $x=0$. The pressure waves generated at the inlet can be seen to be reflected at the symmetry plane.

When the boundary layer reaches $x=0$, it is deflected and a relaminarization process starts. Strong pressure waves are generated and, since the flow at the bump reaches sonic speed, the pressure wave and the outermost 


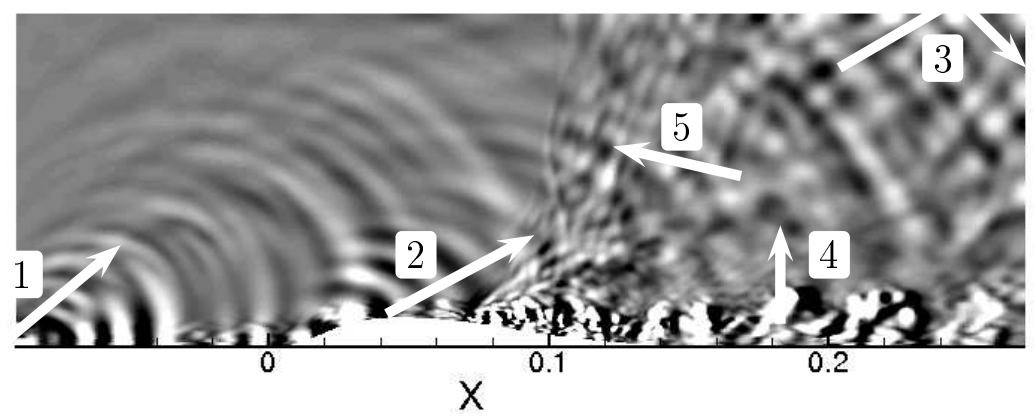

Figure 14: Snapshot of $d p / d t$ for case 2.

part of the convected structures will have the same speed (arrow number 2). Waves with low amplitude seem to be eradicated as they pass through the separation region, and only the outer parts of the wave front remain undisrupted. Waves with high amplitude seem on the other hand to pass through the shock. If the separation produces sound waves, they are either much weaker than the waves from the relaminarization process or coincides with them.

Even though the lower parts of some of the wave fronts are missing, the waves emanating from the relaminarization and the deflection of the flow at $x=0$ will be the dominating pressure waves in the freestream behind the shock. They reach the symmetry plane where they are deflected towards the outlet (arrow number 3).

In accordance with the theory of Handa et al. (2003) pressure waves do reach the upper boundary of the domain. They orginate however not only from the separated region but also from the relaminarization and the deflection of the flow. The shear layer created by the separation is actually rather quiescent and generates only weak pressure waves that propagate out in the freestream behind the shock (arrow number 4). The exact origin of the waves that reach the ceiling will of course depend on the exact geometry of the bump. Handa et al. used a more aggressively curved and thereby shorter bump.

There are also some waves behind the shock that seem to be parallel to it (arrow number 5). They travel slowly upstream and are probably created 


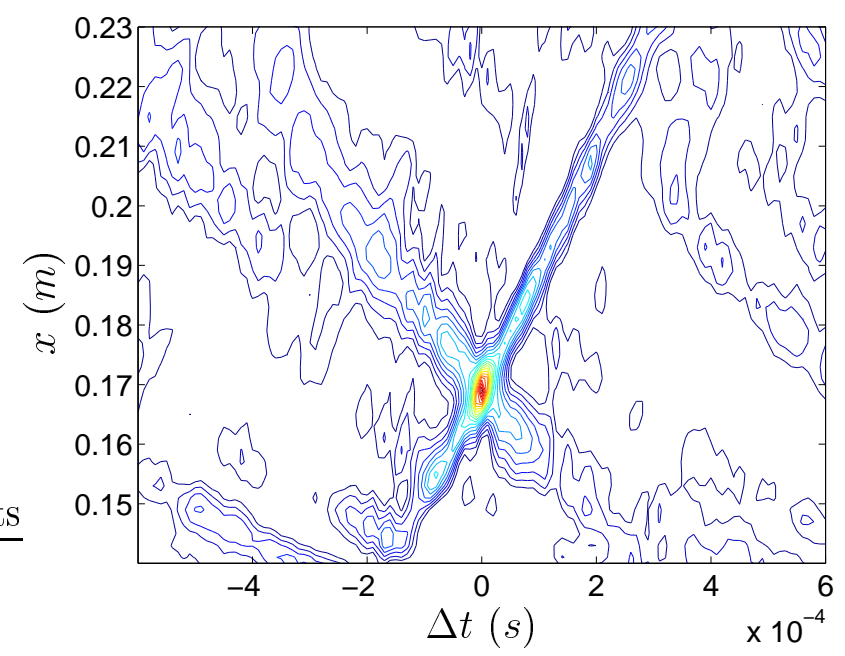

Figure 15: Time-space pressure-pressure correlation for case 4 taken at $y=0.1125$. Contour level difference is 0.05 .

by the fixed pressure boundary condition, which is known to deflect sound waves. The only way they can play an important role in this investigation is if they somehow prevents large scale shock movement. However unlikely that is, it can only be ruled out if an buffer zone is added.

Handa et al. used space-time correlations of pressure fluctuations along the top wall to argue that, when pressure waves from the separation region hit the ceiling, the upper boundary layer is affected and information is brought upstream through the boundary layer to the front of the shock where it would make the shock change position. Figure 15 shows the space-time correlation for the pressure in the upper boundary layer of case 4 . Frequency and phase plots reveal that the plot has one dominating frequency at $1.15 \mathrm{kHz}$ which is traveling downstream. This is supported by the fact that the main ridge in figure 15 has the same inclination as the velocity time-space correlations calculated at the same point. The only important frequency that is traveling upstream by the pressure is the one at $3.9 \mathrm{kHz}$ and that is emanating from further downstream. This frequency is in the turbulent range and is probably only a manifestation of the ellipticity of the equations. Hence, the results presented here do not support the hypothesis of Handa et al. 


\section{Summary and discussion}

Well resolved large eddy simulations of transonic flow with shock wave/turbulent boundary layer interaction have been presented. The quality of the calculations has not been shown in this paper but the issue is thoroughly treated in Wollblad et al. (2006) where the features of a baseline case are discussed in detail. Alterations inspired by other studies have been made both to the domain and to the flow boundary conditions.

Neither the baseline case, nor any of the four new cases presented here display any large scale shock movement. In three of the cases, the lower part of the shock moves. This movement is argued to be related to elliptic leakage. The hypothesis of Handa et al. (2003) is found not to be valid in the present work. There are pressure waves that reach the ceiling but they emanate primarily from the front of the bump and not from the separation region. Only turbulent pressure information seems to travel upstream along the ceiling.

There is of course always room for improvement and further investigation. The authors have strong faith in the discretization scheme used, but testing another scheme would have lent even greater credibility to the calculations. However unlikely it is that the weak sound waves generated at the outlet would hinder shock movement, it can only be excluded if the calculations were made using a buffer zone. Finally a more advanced shock capturing method could be tested.

There is still another interesting theory about large scale shock movement that has not been considered here. Bogar et al. (1983) reported measurements made in the same wind tunnel used by Sajben and Kroutil in their investigation of effects of initial boundary layer thickness (Sajben and Kroutil, 1981). The wind tunnel was not closed but the air was ejected a distance downstream of the sonic throat. By altering the length of the test section, Bogar et al. concluded that, if the test section was long enough for the upper and lower boundary layers to merge, the frequency of the shock movement scaled with the distance from the shock to the merging point. Otherwise, the frequency of the shock movement scaled with the distance from the shock to the outlet. Biswas (2004) used the same domain to make 3D RANS calculations and drew the same conclusion. He added the observation that suction slots in the walls could play the same role as the exit or the merging point of the boundary layers. This theory cannot be confirmed by LES since the computational domain would be much larger than can be handled, at least 
in the near future.

The conclusion drawn in this investigation is that large scale movement of the whole shock is probably not a local phenomenon. Only the movement of the innermost part of the shock might be locally triggered by elliptic leakage. Otherwise, it is likely that large scale shock movement is created by disturbances in experimental facilities or resonance between the shock and some distant part of the flow not included in the simulations presented here.

\section{Acknowledgments}

This project is sponsored by the Swedish Energy Agency and is work carried out in cooperation with the Royal Institute of Technology.

\section{References}

AGARD, 1986. Shock-wave boundary layer interactions. AGARDograph No.280.

Andreopoulos, J., Muck, K. C., 1987. Some new aspects of the shockwave/boundary-layer interaction in compression-ramp flows. AIAA Journal $180,405-428$.

Bachalo, W. D., Johnson, D. A., 1986. Transonic, turbulent boundary-layer separation generated on an axisymmetric flow model. AIAA Journal 24 (3), $437-443$.

Bibko, V., Efimtsov, B., Korkach, V., Kuznetsov, V., 1990. Oscillations of a shock wave induced by boundary layer separation. Fluid Dynamics 25 (4), 639-641.

Biswas, D., 2004. Unsteady 3-d navier-stokes simulations of characteristic frequency and length scales in transonic diffuser. AIAA conference, paper number 2004-2715.

Bodony, D. J., Lele, S. K., 2006. Review of the current status of jet noise predictions using large-eddy simulation. 44th Aerospace Sciences Meeting and Exhibit, aIAA Paper 2006-0468.

Bogar, T. J., Sajben, M., Kroutil, J. C., 1983. Characteristic frequencies of transonic diffuser flow oscillations. AIAA Journal 9, $1232-1240$. 
Bron, O., 2003. Numerical and experimental study of the shock-boundary layer interaction in transonic unsteady flow. Ph.D. thesis, Royal Institute of Technology, Sweden.

Cabana, M., Fortuné, V., Jordan, P., 2006. A look inside the lighthill source term. AIAA conference, paper number 2006-2484.

Deck, S., 2005. Numerical simulation of transonic buffet over a supercritical airfoil. AIAA Journal 43, 1556 - 1566.

Eriksson, L.-E., 1995. Development and validation of highly modular flow solver versions in g2dflow and g3dflow series for compressible viscous reacting flow. Internal report 9970-1162, Volvo Aero Corporation, Sweden.

Glotov, G., 1998. The features of incipiency and development of the recirculated zones in the supersonic layers of supersonic flow. Appl. Mech. Tech. Phys. 36 (5), 30-39, in Russian.

Handa, T., Masuda, M., Matsuo, K., 2003. Mechanism of shock wave oscillation in transonic diffusers. AIAA Journal 41, $64-70$.

Jameson, A., Schmidt, W., Turkel, E., 1981. Numerical solutions of the euler equations by finite volume methods using runge-kutta time-stepping schemes. AIAA paper, paper number 81-1259.

Jones, W. P., Launder, B. E., 1971. The prediction of laminarization with a two-equation model of turbulence. International Journal of Heat and Mass Transfer 15 (2), $301-314$.

Knight, D., Yan, H., Panaras, A. G., Zheltovodov, A., 2003. Advances in CFD prediction of shock wave turbulent boundary layer interactions. Progress in Aerospace Science 39 (4), 121 - 184.

Lee, B. H. K., 1990. Oscillation shock motion caused by transonic shock boundary-layer interaction. AIAA Journal 28, 942 - 944.

Moroianu, D., 2003. Numerical simulations of turbulent flows at widely different mach nubers. Ph.D. thesis, Lund University.

Nicoud, F., Ducros, F., 1999. Subgrid-scale stress modelling based on the square of the velocity gradient tensor. Flow, Turbulence and Combustion $62(3), 183-200$. 
Sajben, M., Kroutil, J. C., 1981. Effects of initial boundary-layer thickness on transonic diffuser flows. AIAA Journal 19, 1386 - 1393.

Sandham, N. D., Yao, Y. F., Lawal, A. A., 2003. Large-eddy simulation of transonic turbulent flow over a bump. International Journal of Heat and Fluid Flow 24 (4), $584-595$.

Thomas, F. O., Putnam, C. M., Chu, H. C., 1994. On the mechanism of unsteady shock oscillation in shock wave/turbulent boundary layer interactions. Experiments in Fluids 18 (1-2), $69-81$.

Wollblad, C., Davidson, L., Eriksson, L.-E., 2006. Large eddy simulation of transonic flow with shock wave/turbulent boundary layer interaction. AIAA Journal 44, 2340 - 2353.

Wollblad, C., Eriksson, L.-E., Davidson, L., 2004. Semi-implicit preconditioning for wall-bounded flow. AIAA conference, paper number 2004-2135.

Wu, M., Martin, M. P., 2004. Direct numerical simulation of shockwave/turbulent boundary layer interaction. AIAA conference, paper number 2004-2145.

Xiao, Q., Tsai, H. M., 2006. Numerical study of transonic buffet on a supercritical airfoil. AIAA Journal 44, 620 - 628. 